\title{
What if the Automation Fails? - A Classification of Scenarios in Teleoperated Driving
}

\author{
Carmen Kettwich \\ German Aerospace Center (DLR), Institute of \\ Transportation Systems, Lilienthalplatz 7, 38108 \\ Braunschweig, Germany \\ carmen.kettwich@dlr.de \\ Hüseyin Avsar \\ German Aerospace Center (DLR), Institute of \\ Transportation Systems, Lilienthalplatz 7, 38108 \\ Braunschweig, Germany \\ hueseyin.avsar@dlr.de
}

\begin{abstract}
Teleoperated driving as an enabler has the potential to bridge the gap to fully automated driving (SAE Level 5 [13]) by monitoring and controlling remotely highly automated vehicles (AVs, SAE 4) whenever their automation fails to do so. To ensure safe and efficient teleoperation, a user-centered human-machine interface (HMI) considering use cases, scenarios, and sequences relevant in teleoperated driving needs to be designed. For this purpose, this paper presents as a grounding an extensive system to classify scenarios relevant to remote-controlled AVs from a control center perspective. It is based on four major categories pertaining to the vehicles, the teleoperation workstation, interaction partners, and the environment. The system will serve as a scaffolding to categorize a catalogue of more than 150 scenarios derived from several research projects and this system will be adapted in future research to fit an ever-broader range of scenarios in the teleoperation of AVs.
\end{abstract}

\section{CCS CONCEPTS}

- Interaction design process and methods; • HCI theory, concepts and models; $\bullet$ Scenario-based design;

\section{KEYWORDS}

HMI, teleoperation interface, automated driving, scenario catalogue

\section{ACM Reference Format:}

Carmen Kettwich, Andreas Schrank, Hüseyin Avsar, and Michael Oehl. 2021. What if the Automation Fails? - A Classification of Scenarios in Teleoperated Driving. In 13th International Conference on Automotive User Interfaces and Interactive Vehicular Applications (AutomotiveUI '21 Adjunct), September 09-14, 2021, Leeds, United Kingdom. ACM, New York, NY, USA, 5 pages. https://doi.org/10.1145/3473682.3480271

Permission to make digital or hard copies of part or all of this work for personal or classroom use is granted without fee provided that copies are not made or distributed for profit or commercial advantage and that copies bear this notice and the full citation on the first page. Copyrights for third-party components of this work must be honored For all other uses, contact the owner/author(s).

AutomotiveUI '21 Adjunct, September 09-14, 2021, Leeds, United Kingdom

(c) 2021 Copyright held by the owner/author(s).

ACM ISBN 978-1-4503-8641-8/21/09.

https://doi.org/10.1145/3473682.3480271

\author{
Andreas Schrank \\ German Aerospace Center (DLR), Institute of \\ Transportation Systems, Lilienthalplatz 7, 38108 \\ Braunschweig, Germany \\ andreas.schrank@dlr.de \\ Michael Oehl \\ German Aerospace Center (DLR), Institute of \\ Transportation Systems, Lilienthalplatz 7, 38108 \\ Braunschweig, Germany \\ michael.oehl@dlr.de
}

\section{INTRODUCTION}

As long as fully automated vehicles (SAE Level 5 [13]) are not entirely feasible, as an enabler, teleoperated driving is on the way to become an important complementary technology with a growing number of pilot projects like the projects this paper is based on, i.e., the European project "CityMobil2" [4] and the German projects "HEAT" [8], "RealLab Hamburg" [11], and "AHEAD" [6]. As soon as the automation reaches its limits, the system of the automated vehicle (AV) transfers the monitoring task from the vehicle to its remote coordinator to be controlled when necessary [2, 7]. In addition to technological developments, a novel user-centered human-machine interface (HMI) for teleoperation needs to be designed in order to maintain traffic safety and efficiency. Within the user-centered design process and development of remote operation workstations, a scenario catalogue forms the basis for deriving user requirements imposed on the control center stations. Therefore, the large number of teleoperated driving scenarios collected requires a generic classification of these scenarios that provides a structured overview without ignoring their complexity. The following paper suggests a classification system to describe scenarios that serve as a basis for the design and evaluation of control center workstations for teleoperated driving, e.g., in the context of public transport [9].

\section{SCENARIOS IN TELEOPERATED DRIVING}

\subsection{Definitions}

Even though typical situations and their embedment into a specific context play a crucial role in the user-centered development of mobility solutions [14], there are no universal definitions of the terms "scene", "scenario", and "use case". For this reason, the terminology used within this paper is based on Ulbrich et al. [14] and Wilbrink et al. [15] and will be defined in the following paragraphs.

A scene describes a snapshot of the environment. It includes a scenery (e.g., lane networks, stationary elements, and environmental conditions), dynamic elements (e.g., dynamic objects' states and attributes), self-representations of actors, and observers (e.g., actors' and observers' states and attributes, skills, and abilities) as well as the relationships between those entities.

A scenario is defined as a temporal development of different scenes within a sequence of scenes. In order to characterize this 
temporal development, events and actions as well as objectives might be specified. Unlike a scene, a scenario describes a period of time. Scenarios start with an initial scene and can be visualized using interaction diagrams (cf. Figure 3).

A use case is a functional description for a technical system and its behavior for a specific use. Use cases can comprise numerous different scenarios, but a scenario can only contain a certain number of scenes arranged in a certain order [14].

For research on human-machine interaction, the focus on singular static scenes does not suffice to describe processes of interaction. On the other side, the system-based level applied in use cases is too abstract to pay enough attention to these processes. Therefore, the focus of this paper will be on scenarios and their classification.

\subsection{Process of Collecting Scenarios}

Participatory observation and expert interviews with control center staff in Hamburg and Braunschweig, Germany, helped examine the working equipment, tasks, roles, and collaborations in a control center for teleoperation in public transport in general.

Next, videos from the EU CityMobil2 project $[1,3]$ were reanalyzed focusing on the interaction of AVs with other road users to generate scenarios. The main objective of CityMobil2 was to implement different demonstrations of AVs in five European cities as a part of local public transport [12]. Then, structured in-depth interviews were carried out with three on-board operators of automated shuttles (SAE Level 4 [13]) integrated in Hamburg's public transport system as part of the HEAT project [11]. These interviews focused on incidents as well as shuttle malfunctions to generate scenarios for teleoperation of AVs. Following summarizing and structuring methods of Mayring's qualitative content analysis approach [10], a systematic classification system for these scenarios was derived and discussed in brainstorming sessions consisting of four researchers. It covers a wide range of different scenarios to be dealt with by teleoperators and describes them generically in order to be suitable for describing scenarios that have not yet been considered.

\section{CLASSIFICATION OF SCENARIOS}

Figure 1 presents an overview of Levels 1 to 3 of the classification of teleoperation scenarios in public transport. The classification system is structured hierarchically. On Level 1, four categories related to teleoperation are specified: (1) vehicle, (2) teleoperation workstation, (3) interaction partner, and (4) environment, described in further detail in section 3.1. Several levels are subordinated to Level 1, the so-called sublevels. These are not mutually exclusive, i.e., several sublevels can be valid for one scenario. Categories are based on the projects mentioned in the introduction.

\subsection{Categories}

The category "Vehicle" consists of the following subcategories (Level 2 categories): (1) technical specifications, indicating details on the vehicle such as vehicle type (shuttle, driveboard, etc.) and the vehicle's level of automation, according to SAE [13], and (2) transported "goods", specifying what the vehicle carries (users, freight, etc.).
The category "Teleoperation Workstation" consists of the following Level 2 categories: (1) roles in teleoperation, indicating what roles are required in the teleoperation center, and (2) task types, specifying what actions have to be conducted by the teleoperator, and (3) the number of vehicles controlled. The specification of the roles is highly dependent on the architecture of the workstation, particularly the type of remote-control applied and the number of vehicles controlled. In any case, a "Remote Coordinator" is needed to oversee operations in a control center for teleoperated driving. If the vehicles are controlled via direct teleoperation, the teleoperator steers, accelerates, and brakes manually similar to driving a car. These tasks are carried out by a "Remote Driving Operator". The "Remote Service Operator" monitors the backend infrastructure of the vehicles and the workstations. The "Service Technician" fixes the vehicle on-site. The task types range from monitoring, communicating, and documenting to give clearance to the vehicle so it can resume its ride and to fully remote-control, either directly as described above or indirectly by drawing trajectories or setting waypoints that the automation uses to calculate waypoints.

The subcategories pertaining to "Interaction Partner" are (1) number and (2) type of interaction partners. Regarding the former, a distinction is made between no interaction partner, a so-called singular scenario, one, that is a bilateral scenario since the teleoperator interacts with one actor, and two or more, i.e., a multilateral scenario. In terms of the type of interaction partners, internal ones are directly associated with the teleoperator. Examples are the teleoperated vehicle, the control center, and its facilities including workstations, on-site mechanical engineers, and the maintenance department. Externally, the classification system includes traffic participants, both motorized, with different vehicle types and levels of automation, and non-motorized ones, which represent most "vulnerable road users", or VRUs [5]. Other external interaction partners are blue light organizations, including the police, firefighters, and emergency services.

Lastly, "Environment" is comprised of (1) traffic environment, (2) infrastructure, and (3) weather situation. The traffic environment encompasses the urbanity level from urban via suburban to rural, the right of way, and the road itself, characterized by its type and condition. Infrastructure refers to facilities relevant to teleoperated driving, e.g., road-site units that convey data from the traffic environment to the vehicle or the teleoperator. Finally, the weather situation may play a role.

\subsection{Exemplary Classification of Scenario}

Figure 2 provides an example for a scenario in teleoperated driving. The described scenario "Emergency Call" is a monitoring task, one of the most central requirements imposed on a teleoperator of vehicles. It is subsumed under the use case "Monitoring" and consists of the elements visualized in Figure 3

Following the system depicted in Figure 1, the scenario is specified. For Level 1 category "Vehicle", e.g., these are the technical specifications such as vehicle type (shuttle) and level of automation (4), and the "goods" transported (user). The other Level 1 categories are structured equally.

The scenario is described in an interaction diagram in Figure 3. It contains the actors involved in the scenario (nodes), their actions 


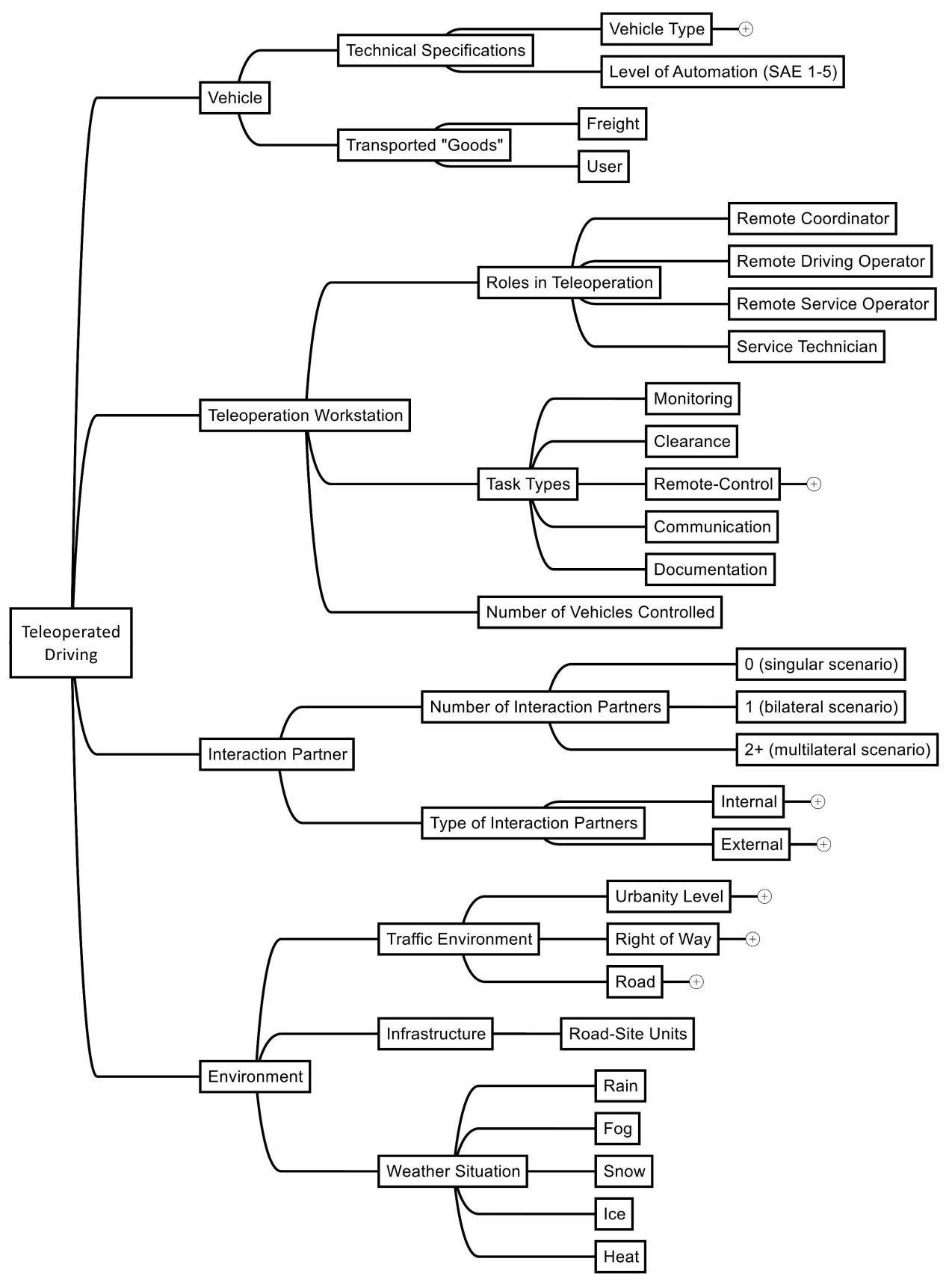

Figure 1: Levels 1-3 of the classification system for scenarios in teleoperated driving. Level 1 categories are "Vehicle", "Teleoperation Workstation", "Interaction Partner”, and "Environment” and entail sublevels. Plus-symbols (+) indicate further sublevels not shown here.

(lines connecting the nodes), and the direction of their actions (arrows). The temporal order of the sequences is delineated by numbers, e.g., 1. If several actions take place simultaneously, letters are attached, e.g., 1a. The diagram will serve as a starting point for analyzing the scenes of the scenario in more detail.

\section{OUTLOOK}

The suggested version of the classification system will help researchers to systematically analyze scenarios in teleoperated driving and derive requirement-based interaction strategies from them. Additionally, it will be used to structure the extensive catalogue of 


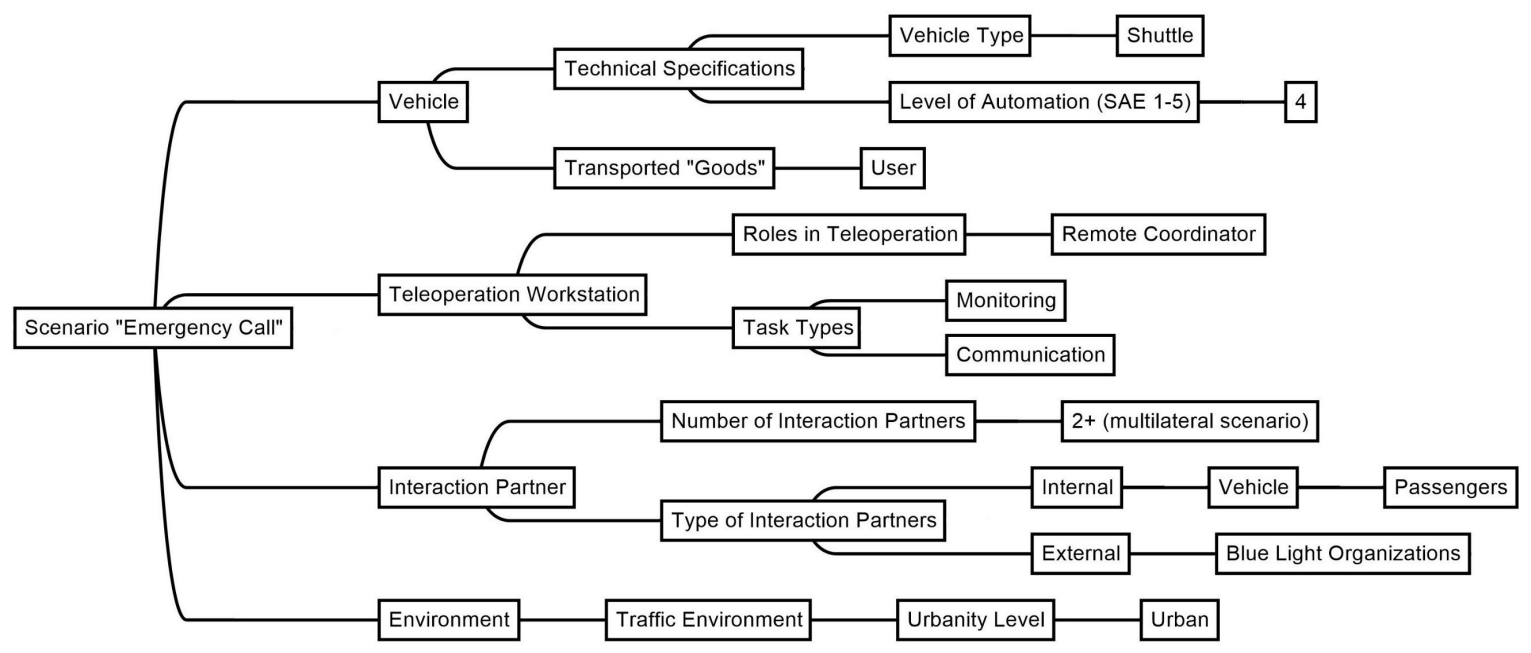

Figure 2: Exemplary classification of scenario "Emergency Call” using the proposed classification system.

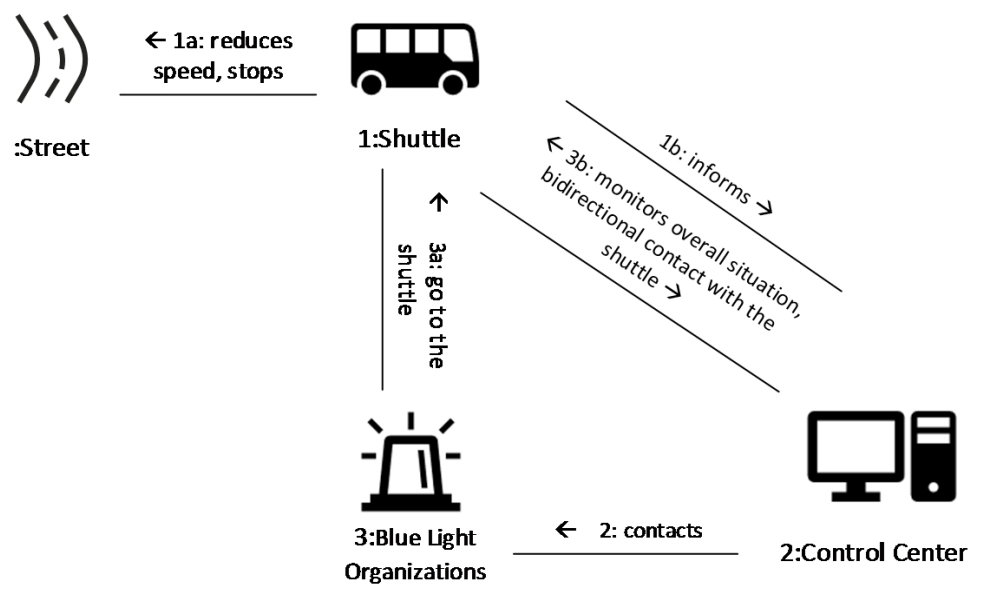

Figure 3: Interaction diagram for scenario "Emergency Call”. It consists of following elements represented in the diagram: The shuttle reduces its speed and stops, if possible, at a safe spot (1a). The shuttle or the passengers inform the control center about the emergency (1b). After that, the control center contacts blue light organizations, such as the medical emergency service, and submits the current position of the shuttle (2). The blue light organizations go to the shuttle and intervene in order to overcome the emergency (3a). In the meantime, control center and passengers are in bidirectional contact with one another, e.g., over a verbal communication line. The control center gets a comprehensive impression of the situation in and around the shuttle based on the video images submitted (3b).

more than 150 scenarios relevant to teleoperated driving that have been collected across the presented projects. During this process, the classification system will be further refined to comprehensively represent all these scenarios and beyond this to help structuring research efforts on scenarios in teleoperation. Upon classification, the scenarios will be validated based on their frequency, safetyrelevance, and other key parameters by experts in mobility research and traffic control center staff, so the most relevant scenarios can be identified und used in further research on teleoperated driving.

\section{REFERENCES}

[1] Adriano Alessandrini, Alessio Cattivera, Carlos Holguin, and Daniele Stam. 2014. CityMobil2: Challenges and Opportunities of Fully Automated Mobility. In Road Vehicle Automation, Gereon Meyer and Sven Beiker, Eds. Springer International Publishing, Cham. https://doi.org/10.1007/978-3-319-05990-7_15 
[2] Frederic Chucholowski, Sebastian Gnatzig, Tito Tang, Amin Hosseini, and Markus Lienkamp. 2013. Teleoperiertes Fahren. Aktuelle Entwicklungen. In 6. Tagung Fahrerassistenz, München.

[3] Marc Dziennus, Anna Schieben, Annika Ilgen, and David Käthner. 2016. How to interact with a Cybercar? - Attitudes and expectations on the interaction and communication with fully automated vehicles. https://doi.org/10.13140/RG.2.2 21819.82726

[4] European Commission. Final Report Summary - CITYMOBIL2 (Cities demonstrating cybernetic mobility). Retrieved July 2, 2021 from https://cordis.europa. eu/project/id/314190/reporting

[5] European Commission. 2021. Intelligent transport systems. ITS \& Vulnerable Road Users. Retrieved July 2, 2021 from https://ec.europa.eu/transport/themes/ its/road/action_plan/its_and_vulnerable_road_users_en

[6] German Aerospace Center. 2020. DLR robotic vehicles will support deliveries in difficult areas for the World Food Programme. Remote-controlled access in crisis regions. Retrieved July 2, 2021 from https://www.dlr.de/content/en/articles/ news/2020/04/20201021_remote-controlled-access-in-crisis-regions.html

[7] Sebastian Gnatzig, Frederic Chucholowski, Tito Tang, and Markus Lienkamp. 2013. A system design for teleoperated road vehicles. ICINCO 2013 - Proceedings of the 10th International Conference on Informatics in Control, Automation and Robotics 2, July, 231-238. https://doi.org/10.5220/0004475802310238

[8] Hamburger Hochbahn 2021. The future is driverless. Be part of the HOCHBAHN research and development project HEAT. Retrieved June 29, 2021 from https://www.hochbahn.de/hochbahn/hamburg/en/home/projects/ expansion_and_projects/project_heat
[9] Carmen Kettwich, Andreas Schrank, and Michael Oehl. 2021. Teleoperation of Highly Automated Vehicles in Public Transport: User-Centered Design of a Human-Machine Interface for Remote-Operation and Its Expert Usability Evaluation. MTI 5, 5, 26. https://doi.org/10.3390/mti5050026

[10] Philipp Mayring. 2000. Qualitative Inhaltsanalyse. Grundlagen und Techniken (7th). Deutscher Studien Verlag, Weinheim.

[11] RealLab Hamburg. 2020. Autonomes Fahren. Retrieved March 25, 2021 from https://reallab-hamburg.de/projekte/autonomes-fahren/

[12] Anna Schieben, Marc Wilbrink, Carmen Kettwich, Ruth Madigan, Tyron Louw, and Natasha Merat. 2019. Designing the interaction of automated vehicles with other traffic participants: design considerations based on human needs and expectations. Cognition, Technology and Work 21, 1, 69-85. https://doi.org/10. 1007/s10111-018-0521-z

[13] Society of Automotive Engineers. 2018. SAE J 3016-201806. Taxonomy and Definitions for Terms Related to Driving Automation Systems for On-Road Motor Vehicles, Washington, D.C. Retrieved July 2, 2021 from https://www.sae.org/ standards/content/j3016_201806

[14] Simon Ulbrich, Till Menzel, Andreas Reschka, Fabian Schuldt, and Markus Maurer. 2015. Defining and Substantiating the Terms Scene, Situation and Scenario for Automated Driving. In IEEE International Annual Conference on Intelligent Transportation Systems, 982-988. https://doi.org/10.1109/ITSC.2015.164

[15] Marc Wilbrink, Anna Schieben, Robert Markowski, Florian Weber, Tina Gehb, Johannes Ruenz, Fabio Tango, Marc Kaup, Jan-Henning Willrodt, Villy Portouli, Natasha Merat, Ruth Madigan, Gustav Markkula, Richard Romano, Charles Fox, Matthias Althoff, Sebastian Söntges, and André Dietrich. 2018. interACT D1.1. Definition of interACT use cases and scenarios. 